\title{
Phenetic distances in the Drosophila melanogaster-subgroup species and oviposition-site preference for food components
}

\author{
M. Bos \& A. Boerema \\ Department of Genetics, University of Groningen, Biological Centre, Kerklaan 30, 9751 NN Haren (Gr.), \\ The Netherlands
}

\begin{abstract}
Oviposition-site preferences (O.S.P.) have been investigated in females of six sibling species of the Drosophila melanogaster subgroup. O.S.P. were determined for standard food components and yeast genotypes. Females of all species showed a strong preference for complete medium and avoidance of pure agar as an egg-deposition site.

Ecological trees of the species on the basis of rank correlations were constructed. In 'no-choice' situations they agree with phylogenetic trees obtained by different means but in 'choice' situations they do not agree too well.

All species showed a high egg production on live yeast compared with standard medium (with killed yeast) and $D$. erecta females demonstrated discrimination between yeast genotypes. Niche breadth calculated from survival on the sterol mutant yeasts correlated fairly well with phylogenetic trees.
\end{abstract}

\section{Introduction}

Recently Lemeunier \& Ashburner (1976) and Eisses et al. (1979) elucidated the phylogenetic relationships between six sibling species of the Drosophila melanogaster subgroup (see e.g. Tsacas \& David, 1974; Tsacas \& Lachaise, 1974) by means of two different approaches, the first based on chromosomal rearrangements and the other on allozyme patterns. Both phylogenetic trees show a considerable similarity. D. melanogaster, D. simulans - domestic cosmopolitan species - and $D$. mauritiana, known from the island of Mauritius (Tsacas \& David, 1974; David \& Tsacas, 1975) are genetically very similar. $D$. erecta, $D$. teissieri and D. yakuba are African endemic species and genetically separated from the above three species (see Fig. 1). The distribution areas of some of the species do overlap. D. mauritiana occurs isolated on the island of Mauritius, while erecta seems to be restricted to the Ivory Coast and Congo in West

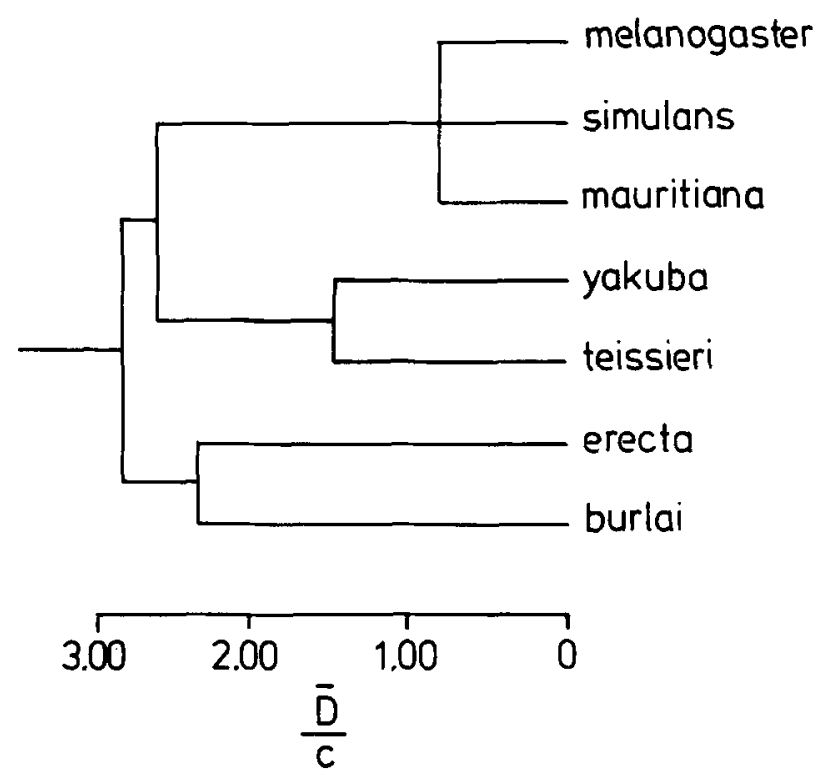

Fig. I. A phylogenetic tree of seven species of the melanogaster species group: six species belonging to the melanogaster subgroup (melanogaster, simulans, mauritiana, lakuba, teissieri and erecta) and one species (burlai) belonging to the montium subgroup (after Eises et al., 1979). 
Africa. In spite of reproductive isolation and thus separate evolution, the sibling species have remained morphologically extremely similar, which was also noticed for species in the $D$. willistoni group (Ayala et al., 1975). Dobzhansky (1956) speculated that the evolution of external morphology in the genus Drosophila has reached a high degree of perfection and that the adaptive evolution proceeds largely through physiological channels.

When closely related species occur in the same area, one would like to know how they specialized. To obtain evidence about species specialization it might be useful to compare the species for developmental, ecological or behavioural characters. High specificity in the choice of the niche in which the females deposit their eggs (Carson, 1971) might be one informative character in this respect as one of the essential components of reproduction.

Live yeast is a component of the environment clearly relevant to Drosophila (da Cunha, 1951; Thoday, 1972; Begon, 1973; Ali \& El Helw, 1974). Cage populations provided with a variety of yeasts and media maintain a higher degree of heterozygosity for enzyme variants than cages providing a more uniform environment in these respects (Powell, 1971; McDonald \& Ayala, 1974). Further Clark et al. (1979) have emphasized the importance of the amount of yeast for the regulation of the quantity of the enzyme alcohol dehydrogenase in $D$. melanogaster.

In the present study we compare the preferences of adult females of the six sibling species to oviposit (O.S.P.'s) on five genotypes of the yeast Saccharomyces cerevisiae and correlate these O.S.P.'s with the larval capability to utilize these yeasts. The five yeast genotypes differ in their sterol contents and are known to support differentially the development of Drosophila species (Bos et al., 1976). Additionally we measure the female preferences for standard food components like sugar and yeast. These comparisons are discussed in relation to genetic distances of the species and niche differentiation.

\section{Material and methods}

\section{Yeast genotypes}

The yeast strains used were a wildtype strain of
Saccharomyces cerevisiae (erg- + ) and four sterol mutants (erg-2, erg-3, erg-5 and erg-6) derived from the A184A wildtype stock described in Bos et al. (1976). The mutants are isogenic with the wildtype except at the relevant erg locus.

\section{Drosophila stocks}

The six species of the Drosophila melanogaster subgroup used in this study - all originated from Africa and were kindly provided by Dr L. Tsacas or Dr W. van Delden. The strains used are listed below. Details about the origin and the year from which on they were kept by mass culture in the laboratory are given in parentheses. All strains were founded with several inseminated females. The numbers refer to the strain numbers of the collection at C.N.R.S., Gif-sur-Yvette, France, Details about the latter strains can be found in Lemeunier \& Ashburner (1976).

D. melanogaster (Seronera, Tanzania; 1973); simulans (Uganda; 1973); mauritiana 163.1 (Mauritius; 1973); yakuba 131.6 (Gabon; 1970); teissieri 144.4 (Cameroun; 1970); erecta 154.1 (Ivory Coast; 1971). The fraction of polymorphic loci in these strains was respectively: $11 / 17,9 / 17,4 / 17,10 / 17$, 10/17 and 4/17 (Van Delden, personal communication; based on the isozyme study reported in Eisses et al., 1979).

\section{Experimental procedures}

Yeast strains were grown and growth tests of Drosophila larvae were made as described in Bos et al. (1976). The Drosophila strains were cultured on a killed yeast medium (see Bos \& Scharloo, 1974), called hereafter 'standard' or 'complete' medium.

\section{Egg production of the Drosophila species}

Daily egg production was determined for one to twenty day old females by counting every 24 hours the total production of ten lids with standard medium, attached to $50 \mathrm{ml}$ bottles with 7-10 unetherised pairs of flies each.

Oviposition site preference ('O.S.P.') on standard food components

Five different media were presented to ten pairs of flies (five up to ten days old) in 50-ml bottles, each containing two slices of medium. The media 
were: $\mathrm{A}=\operatorname{agar}(2 \%) ; \mathrm{E}=\operatorname{agar}$ with ethanol $(1 \%) ; \mathrm{Y}$ $=$ agar with killed baker's yeast $(3.2 \%) ; \mathrm{S}=$ agar with sucrose $(5.4 \%)$; $\mathrm{C}=$ complete medium, with all the components in the given percentages. $\mathrm{E}$ and $\mathrm{C}$ contained also nipagine as in the standard medium. The flies were given 24 hours to lay eggs at $25 \pm$ $0.5^{\circ} \mathrm{C}$ and $60-80 \%$ R.H. on the two slices of medium. In the 'no-choice' situation the two slices were of the same medium, in the 'choice' situation the two slices were of different medium: all possible combinations of media were presented and every test was made with five replicates. All tests with one species were done at the same time.

\section{'O.S.P.' on yeast genotypes}

'O.S.P.' of the six Drosophila species were also measured in a yeast choice experiment in population cages (Beardmore et al., 1963). In three cages (per species) the tops of fifteen rubber stops put in the holes were covered with a $5 \mathrm{~mm}$ agar (2\%) layer, coated with fresh live yeast. Each cage contained three replicates of each of the five Saccharomyces cerevisiae genotypes. In one of the cages the position of the sites was constant over the three experimental days, in two of the cages the positions were changed every day. Every cage contained 200 pairs of five to ten days old flies. Egg production (in 24 hours) was measured on three successive days.

As a control a cage with fifteen replicates of standard medium was presented to each of the six Drosophila species. All experiments were carried out between June 1976 and June 1979.

\section{Results}

\section{Egg production}

In Figure 2, the daily egg production of the six Drosophila species is presented for theirfirst twenty days of life. All the species reach maximum production per day within two to five days, stay for about six days on a plateau and production declines after the tenth day. For the oviposition site experiments it is important to know if the species differ greatly in their production during the test days (the females

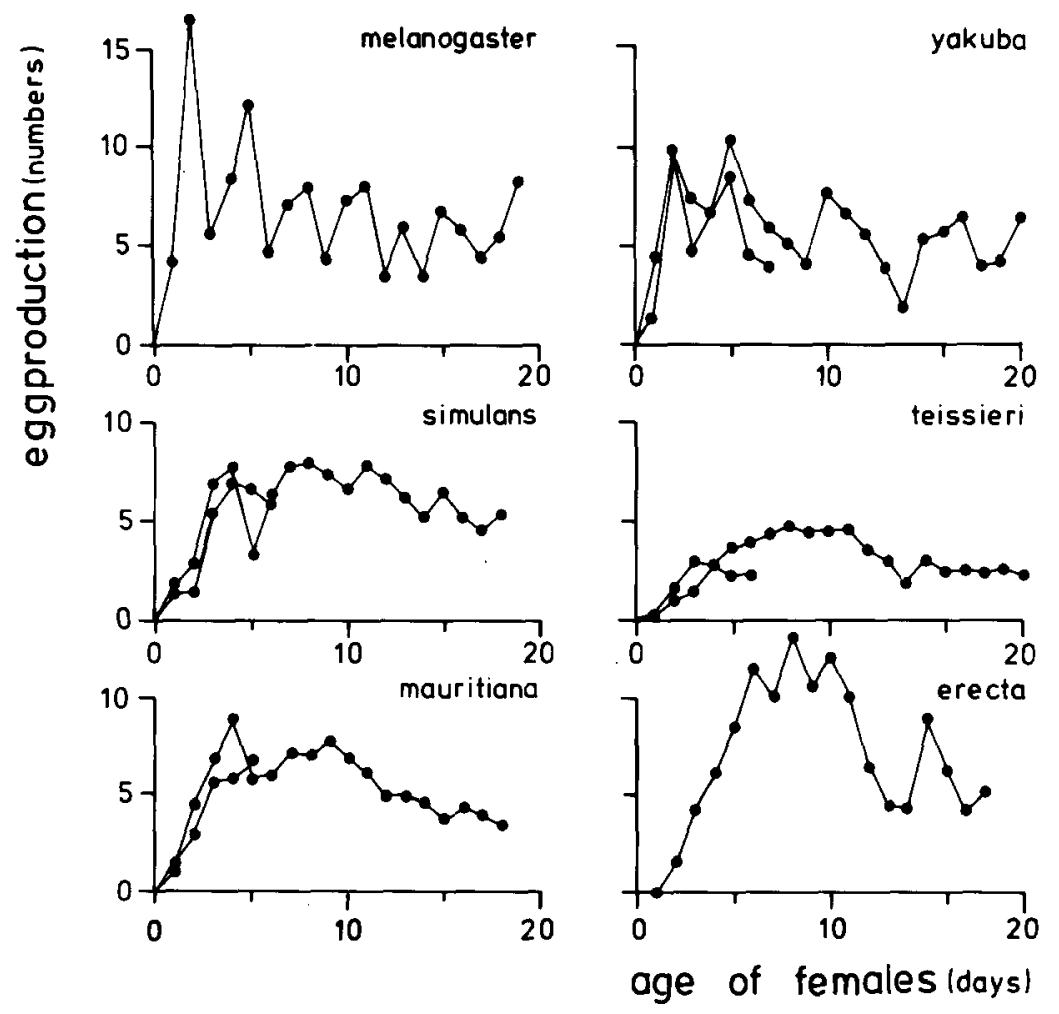

Fig. 2. Daily egg production of the six D. melanogaster-subgroup species (number of eggs per female). 
used were five to ten days old). All the species do have a relatively high production during the test days. D. melanogaster and yakuba reach their maximal production within two days. A smaller experiment with four of the species (included in Fig. 2) confirms these conclusions.

\section{Oviposition site preferences ('O.S.P.')}

\section{On standard food components}

In Table 1 mean egg production \pm standard error is presented for all the test situations. Figure 3 shows a summary of the data from Table 1. Comparison of the 'averages' in this table clearly shows a higher production on complete (C) medium than on agar (A) and ethanol (E) for all the species.

In the 'choice' and the 'no-choice' tests all the species prefer $\mathrm{C}$ medium and avoid $\mathrm{A}$. On $\mathrm{E}$ melanogaster and simulans have a high production in the 'no-choice' case, which disappears in the 'choice' tests. D. erecta and teissieri restrict their

Table I. Egg production/slice of medium $(\overline{\mathrm{x}} \pm$ s.e.) on five different food components in the presence of the same or an other component (C, S, Y, E, or A). 'Average' is the average production on a medium in the 'choice' situations. Production on e.g. 'Complete $S$ ' means egg production on complete medium in the presence of sugar. This is the complement of 'Sugar C', which means egg production on the sugar medium in the presence of complete medium. The data of the 'no-choice' experiments are presented under 'Complete $C$ ', 'Yeast $Y$ ' etc.

\begin{tabular}{|c|c|c|c|c|c|c|c|}
\hline & & & & ecies & & & \\
\hline Producti & on on: & melanogaster & simulans & mauritiana & yakuba & teissieri & erecta \\
\hline Complet & e $\mathrm{C}$ & $8.4 \pm 1.7$ & $8.0 \pm 2.5$ & $7.0 \pm 0.7$ & $18.4 \pm 2.9$ & $12.6 \pm 1.5$ & $10.6 \pm 2.1$ \\
\hline & $\mathbf{S}$ & $18.0 \pm 5.3$ & $36.6 \pm 11.1$ & $26.2 \pm 1.8$ & $18.0 \pm 2.4$ & $10.4 \pm 0.7$ & $10.4 \pm 5.1$ \\
\hline & $Y$ & $22.4 \pm 6.3$ & $20.2 \pm 3.0$ & $16.8 \pm 3.9$ & $29.8 \pm 1.3$ & $20.0 \pm 2.5$ & $1.4 \pm 1.0$ \\
\hline & $\mathrm{E}$ & $17.4 \pm 6.0$ & $14.0 \pm 2.0$ & $24.8 \pm 9.2$ & $18.8 \pm 2.2$ & $17.0 \pm 2.6$ & $3.4 \pm 1.6$ \\
\hline & A & $6.6 \pm 0.9$ & $34.2 \pm 8.6$ & $22.6 \pm 4.9$ & $13.2 \pm 2.7$ & $12.8 \pm 2.9$ & $9.2 \pm 8.4$ \\
\hline average & & $16.1 \pm 3.4$ & $26.3 \pm 5.5$ & $22.6 \pm 2.1$ & $20.0 \pm 3.5$ & $15.1 \pm 2.1$ & $6.1 \pm 2.2$ \\
\hline Yeast & $\mathrm{C}$ & $0.4 \pm 0.2$ & $1.8 \pm 0.4$ & $8.6 \pm 2.5$ & $8.6 \pm 3.3$ & $0.4 \pm 0.2$ & $0.6 \pm 0.2$ \\
\hline & $\mathrm{S}$ & $4.0 \pm 1.7$ & $3.6 \pm 0.9$ & $10.4 \pm 1.4$ & $6.8 \pm 1.5$ & $0.6 \pm 0.6$ & $0.4 \pm 0.4$ \\
\hline & $Y$ & $2.1 \pm 0.6$ & $5.2 \pm 0.8$ & $4.1 \pm 0.7$ & $3.1 \pm 0.7$ & $0.1 \pm 0.1$ & $1.6 \pm 1.1$ \\
\hline & $\mathrm{E}$ & $3.8 \pm 2.0$ & $14.8 \pm 5.1$ & $6.4 \pm 0.9$ & $1.4 \pm 0.9$ & $1.0 \pm 0.8$ & $3.0 \pm 1.1$ \\
\hline & A & $4.2 \pm 1.4$ & $7.8 \pm 1.9$ & $2.8 \pm 1.2$ & $2.0 \pm 0.8$ & $1.0 \pm 0.8$ & $1.0 \pm 0.6$ \\
\hline average & & $3.1 \pm 0.9$ & $7.0 \pm 2.9$ & $7.1 \pm 1.6$ & $4.7 \pm 1.8$ & $0.8 \pm 0.2$ & $1.3 \pm 0.6$ \\
\hline Sugar & $\mathrm{C}$ & $13.6 \pm 2.3$ & $15.6 \pm 6.7$ & $6.2 \pm 1.4$ & $2.4 \pm 0.8$ & $7.8 \pm 3.4$ & $8.4 \pm 2.0$ \\
\hline & $\mathrm{S}$ & $2.4 \pm 0.7$ & $5.2 \pm 1.3$ & $8.5 \pm 1.9$ & $2.5 \pm 2.3$ & $8.1 \pm 1.4$ & $2.9 \pm 1.3$ \\
\hline & $Y$ & $13.0 \pm 5.1$ & $10.4 \pm 2.6$ & $3.0 \pm 2.0$ & $14.4 \pm 1.7$ & $9.4 \pm 4.3$ & $11.2 \pm 2.9$ \\
\hline & $\mathrm{E}$ & $6.0 \pm 0.9$ & $13.6 \pm 2.3$ & $3.2 \pm 1.8$ & $3.6 \pm 2.2$ & $7.8 \pm 1.4$ & $9.4 \pm 1.6$ \\
\hline & A & $11.2 \pm 2.8$ & $4.0 \pm 2.0$ & $4.8 \pm 2.7$ & $4.8 \pm 2.1$ & $14.8 \pm 2.6$ & $2.0 \pm 1.1$ \\
\hline average & & $11.0 \pm 1.7$ & $10.9 \pm 2.5$ & $4.3 \pm 0.8$ & $6.3 \pm 2.7$ & $10.0 \pm 1.7$ & $7.8 \pm 2.0$ \\
\hline Ethanol & $\mathrm{C}$ & $1.0 \pm 0.8$ & 0.0 & $10.8 \pm 3.2$ & 0.0 & $0.4 \pm 0.2$ & $0.6 \pm 0.4$ \\
\hline & $s$ & $3.8 \pm 1.9$ & $0.6 \pm 0.4$ & $5.4 \pm 0.9$ & $0.2 \pm 0.2$ & 0.0 & $0.2 \pm 0.2$ \\
\hline & $\mathrm{Y}$ & $4.8 \pm 1.9$ & $2.0 \pm 1.1$ & $1.4 \pm 0.4$ & $0.4 \pm 0.2$ & 0.0 & $0.6 \pm 0.4$ \\
\hline & $\mathrm{E}$ & $7.2 \pm 2.6$ & $6.7 \pm 1.8$ & $0.8 \pm 0.4$ & $0.5 \pm 0.5$ & $0.3 \pm 0.4$ & $0.2 \pm 0.1$ \\
\hline & A & $1.4 \pm 0.7$ & $0.4 \pm \quad 0.4$ & $2.0 \pm 1.4$ & 0.0 & $0.2 \pm 0.2$ & $0.8 \pm 0.4$ \\
\hline average & & $2.8 \pm 0.9$ & $0.8 \pm 0.4$ & $4.9 \pm 2.2$ & $0.2 \pm 0.1$ & $0.2 \pm 0.1$ & $0.6 \pm 0.1$ \\
\hline Agar & $\mathrm{C}$ & $3.0 \pm 2.5$ & $0.4 \pm 0.2$ & $0.2 \pm 0.2$ & $1.0 \pm 0.6$ & $0.4 \pm 0.2$ & 0.0 \\
\hline & $S$ & $2.2 \pm 1.6$ & 0.0 & $1.6 \pm 1.6$ & $0.2 \pm 0.2$ & $0.2 \pm 0.2$ & $0.4 \pm 0.4$ \\
\hline & $Y$ & $3.8 \pm 1.8$ & $0.2 \pm 0.2$ & $4.4 \pm 1.5$ & 0.0 & 0.0 & 0.0 \\
\hline & $\mathrm{E}$ & $0.8 \pm 0.4$ & $0.4 \pm \quad 0.4$ & $1.6 \pm 0.5$ & $0.4 \pm 0.4$ & $0.2 \pm 0.2$ & $1.0 \pm 0.3$ \\
\hline & A & $1.2 \pm 0.4$ & $0.6 \pm \quad 0.2$ & $0.7 \pm 0.4$ & $0.1 \pm 0.1$ & $0.1 \pm 0.1$ & $0.3 \pm 0.2$ \\
\hline average & & $2.5 \pm 0.6$ & $0.3 \pm 0.1$ & $2.0 \pm 0.9$ & $0.4 \pm 0.2$ & $0.2 \pm 0.1$ & $0.4 \pm 0.2$ \\
\hline
\end{tabular}


NO CHOICE

erecta
teissieri
yakuba
mauritiana
simulans
melanogaster

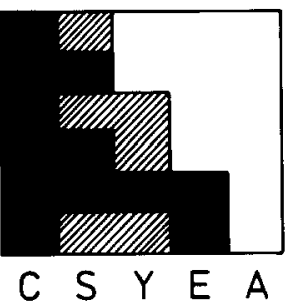

Fig. 3. Oviposition on five media: C, S, Y, E and A (see Materials and methods).

$\square$ no production of eggs ( $<3$ eggs per slice)

low production (3-5 eggs)

high production $>5$ eggs)

production to $\mathrm{C}$ and $\mathrm{S}$. So, they oviposit only if sugar is present. On the whole erecta and teissieri females seem to behave as 'specialists': they use only three or four of the ten sites, while melanogaster, simulans and mauritiana behave like 'generalists' and use seven or eight of the ten sites; yakuba six (Fig. 3).

\section{On yeast genotypes}

To investigate female discrimination between yeast genotypes, four sterol mutant strains and one wildtype yeast strain were presented together in population cages. As a control egg production was measured in cages with standard medium.

Using the egg productions on the fifteen sites in the control cages during three successive days, Spearman's rank correlations $\left(r_{s}\right)$ were calculated between day productions of all the species. None of the species shows position preferences within cages $\left(-0.44<r_{s}<+0.32\right)$. A within-species analysis of

variance was made to examine the presence of effects of cage, day of oviposition and yeast genotype. The significance of main effects and interactions are presented in Table 2. From these anovas it is clear that in all the species except $D$. erecta, there are no cage or yeast effects and all the species show a significant oviposition-day (B) effect. In the exceptional interactions which are significant, the day effect is involved. The effect of day on egg production is clearly show in Table 3 . On the first day the egg production is much lower than on the two following days. The difference between days is more evident in the cages with live yeast than in the cages with complete, standard medium. Table 3

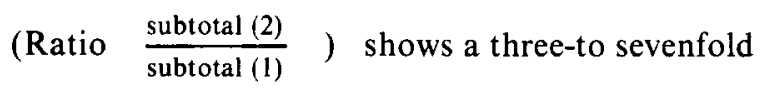
egg production on live yeast compared to standard medium with killed yeast. This higher egg production is present from the first day of testing (see Ratio on day 1, Table 3), in an extreme form in melanogaster and erecta. The ratio of the egg productions on live yeast and complete medium is highest in $D$. melanogaster and simulans. The discrimination between the two media is smallest in mauritiana and teissieri. Total egg productions of melanogaster, simulans and yakuba are high while that for teissieri is low (see also Fig. 1).

The anova (Table 2) indicates a yeast effect for $D$. erecta females only. In the three cages erecta females produce the following percentages of eggs (mean \pm s.e.; expressed in angles) on the five different yeast genotypes:

$$
\begin{array}{ccccc}
\text { erg-6 } & \text { erg-2 } & \text { erg-5 } & \text { erg-t } & \text { erg-3 } \\
21.3 \pm 1.0 & 24.6 \pm 0.9 & 25.1 \pm 2.0 & 27.3 \pm 0.9 & 33.1 \pm 2.1
\end{array}
$$

Table 2. Analyses of variance: significance of effects in the oviposition site preferences experiments on sterol mutant yeasts.

\begin{tabular}{lllllll}
\hline $\begin{array}{l}\text { Main effects } \\
\text { Cage effect (A) }\end{array}$ & melanogaster & simulans & mauritiana & yakuba & teissieri & erecta \\
Day effect (B) & $* * *$ & n.s. & n.s. & n.s. & n.s. & $* *$ \\
Yeast effect (C) & n.s. & $* *$ & $* * *$ & $* * *$ & $* * *$ & $* *$ \\
& & n.s. & n.s. & n.s. & n.s. & $* * *$ \\
$\begin{array}{l}\text { Interactions: } \\
\text { AB }\end{array}$ & & & & & \\
BC & n.s. & n.s. & $* * *$ & n.s. & n.s. & $*$ \\
AC & n.s. & n.s. & n.s. & $*$ & n.s. & n.s. \\
ABC & n.s. & n.s. & n.s. & n.s. & n.s. & n.s. \\
\hline
\end{tabular}

n.s. $=$ non-significant; ${ }^{*}=\mathrm{P}<0.05 ; * *=\mathrm{P}<0.02 ;{ }^{* *}=\mathrm{P}<0.01$. 
Table 3. Number of eggs/day/cage.

\begin{tabular}{|c|c|c|c|c|c|c|c|}
\hline \multirow{2}{*}{ Medium } & \multicolumn{7}{|c|}{ Species } \\
\hline & melanogaster & simulans & mauritiana & yakuba & teissieri & erecta & Total \\
\hline \multicolumn{8}{|l|}{ Complete medium } \\
\hline day 1 & 82 & 298 & 265 & 361 & 195 & 203 & 1404 \\
\hline day 2 & 430 & 227 & 353 & 612 & 152 & 327 & 2101 \\
\hline day 3 & 348 & 416 & 469 & 673 & 272 & 579 & 2757 \\
\hline Subtotal (1) & 860 & 941 & 1087 & 1646 & 619 & 1109 & 6262 \\
\hline \multicolumn{8}{|c|}{ Live Yeast Medium } \\
\hline day 1 & 557 & 970 & 287 & 900 & 243 & 863 & 3820 \\
\hline day 2 & 2858 & 2068 & 1460 & 2335 & 596 & 1774 & 11091 \\
\hline day 3 & 2460 & 2433 & 1468 & 2484 & 704 & 2213 & 11762 \\
\hline Subtotal (2) & 5875 & 5471 & 3215 & 5719 & 1543 & 4850 & 26673 \\
\hline Total & 6735 & 6412 & 4302 & 7365 & 2162 & 5959 & 32935 \\
\hline Ratio $\frac{\text { Subtotal (2) }}{\text { Subtotal (1) }}$ & 6.8 & 5.8 & 3.0 & 3.8 & 2.5 & 4.4 & 5.3 \\
\hline Ratio on day $1^{*}$ & 6.8 & 3.3 & 1.1 & 2.5 & 1.2 & 4.3 & 2.7 \\
\hline
\end{tabular}

* Number of eggs on 'live-yeast medium' day I, divided by number of eggs on 'complete medium' day 1.

Lines under the data connect the yeasts which are not significantly different using Tukey's multiple comparisons test ( $5 \%$ level). It can be seen, that $D$. erecta females seem to discriminate $\mathrm{erg}-3$ from erg6, erg-2 and erg-5.

\section{Growth tests}

The egg-to-pupa and egg-to-adult survival of the six subgroup species on the five yeast genotypes were measured. In Figure 4 the survival is presented. None of the species survives on erg-6. D. mauritiana, yakuba and erecta show normal (compared with survival on wildtype yeast) egg-to-pupa survival on erg-2, teissieri is intermediate. D. melanogaster and simulans have very low survival. No apparent differences within species are seen on erg3 , erg-5 and +-yeast. During the pupal phase all the individuals of melanogaster, simulans and teissieri, grown on erg-2, die. D. yakuba and erecta show low, and mauritiana normal, egg-to-adult survival on this yeast genotype.

Thus on average only survival on erg- 2 discriminates clearly between the six species.

\section{Discussion}

During the evolution of the Drosophila melano-

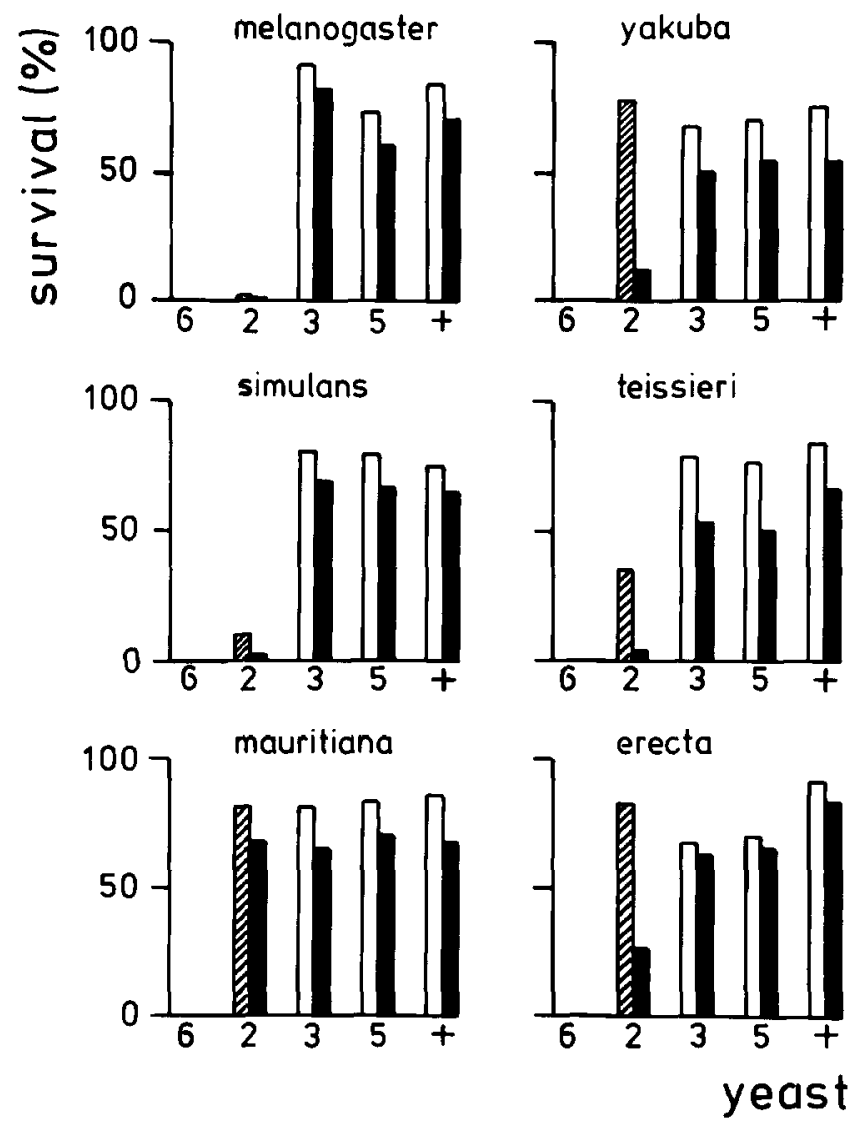

Fig. 4. Survival (\%) of the six sibling species on five yeast genotypes. White: egg-to-pupa survival. Black: egg-to-adult survival. Hatched: egg-to-pupa survival on erg-2. 
gaster subgroup selection for avoidance of competition may have increased the ecological divergence of the species (Parsons, 1973, p. 175). This divergence may have taken place in the larval or the imaginal stage or in both. The larvae may have been selected for differential survival on different feeding sites. The adults may have been differentiated in courtship or breeding behavior.

In our present study we looked at one larval aspect: the development on five yeast genotypes and at one aspect of adult breeding behaviour: oviposition site preferences. We compared African populations only, which were started from several inseminated females, kept in mass bred cultures and in which the fraction of polymorphic loci was between $4 / 17$ and $11 / 17$.

In three out of four experiments we used egg production as the character to compare the sibling species. From the experiment in which we determined egg production of the six sibling species during the first twenty days of life, it is clear that during our experiments females (5-10 days old) had high production in all the species (Fig. 2). The smaller experiment with four of the species (included in Fig. 2) confirmed these facts.

One character of a species which specifies its ecological divergence is the niche breadth (Levins, 1968). We calculated niche breadth for the four comparisons of the species. This is presented in Table 4. For egg production on five yeast oviposition sites (column 1) niche breadth is high and not different between the species, which means that the species did not distinguish between the five yeast genotypes. The yeast genotypes differ only in sterol contents; so, the Drosophila species did not dis- criminate between these biochemical differences between yeast strains. Only $D$. erecta showed a yeast effect. The females strongly prefer $\mathrm{erg}-3$ yeast. Niche breadth calculated from the survival on the sterol mutant yeasts (Table 4 - column 2) does reveal species differences. The range of the species correlates with the phylogenetic relations, established by Lemeunier \& Ashburner (1976) and Eisses et al. (1979): melanogaster close to simulans, teissieri close to yakuba. An exception is D. mauritiana. This is mainly because this species, in contrast with the others, had good survival on the erg-2 yeast.

Columns 3 and 4 of Table 4 show that females of the species clearly differed in oviposition-site preference for standard medium food components, mostly in the 'no-choice' (column 3) situation (see also Table 1 and Fig. 3). In this situation we find that the two cosmopolitan species simulans and melanogaster and mauritiana score with a relatively broad niche compared with teissieri especially with yakuba and erecta. Females of the last two species seem to behave as specialists. The sequence of the species again shows a good correlation with the phylogenetic relation mentioned earlier. This in contrast to the 'choice' situation (column 4). In these 'choice' experiments all the species still prefer the complete medium slices (see also Table 1 and Fig. 3); melanogaster and simulans lose their high production on ethanol, which was also found by McKensie \& Parsons (1972).

The range of the niche breadth is larger in the 'no-choice' situation (0.83-0.34), compared to the 'choice' experiment (0.63-0.43). This decrease of the differences between species in the 'choice'

Table 4. Niche breadth (B) in Drosophila melanogaster subgroup-species in 4 experiments.

\begin{tabular}{|c|c|c|c|c|c|c|c|}
\hline \multicolumn{2}{|c|}{$\begin{array}{l}\text { (1) Egg production } \\
\text { on yeast oviposi- } \\
\text { tion sites } \\
\text { (with choice) }\end{array}$} & \multicolumn{2}{|c|}{$\begin{array}{l}\text { (2) Survival } \\
\text { on sterol } \\
\text { mutant yeasts }\end{array}$} & \multicolumn{2}{|c|}{$\begin{array}{l}\text { (3) Egg production } \\
\text { on aviposition } \\
\text { sites } \\
\text { (no choice) }\end{array}$} & \multicolumn{2}{|c|}{$\begin{array}{l}\text { (4) Egg production } \\
\text { on oviposition } \\
\text { sites } \\
\text { (with choice) }\end{array}$} \\
\hline mel. & 0.99 & maur. & 0.80 & sim. & 0.82 & mel. & 0.63 \\
\hline $\operatorname{sim}$ & 0.98 & erecta & 0.80 & mel. & 0.68 & maur. & $0.5 j$ \\
\hline maur. & 0.98 & yak. & 0.74 & maur. & 0.65 & erecta & 0.52 \\
\hline yak. & 0.98 & teis. & 0.73 & teis. & 0.50 & $\operatorname{sim}$ & 0.48 \\
\hline teis. & 0.97 & sim. & 0.63 & erecta & 0.39 & yak. & 0.44 \\
\hline erecta & 0.96 & mel. & 0.59 & yak. & 0.34 & teis. & 0.43 \\
\hline
\end{tabular}

$\mathrm{B}=1 / \mathrm{n} \Sigma_{\mathrm{p}_{i}}{ }^{2}\left(\mathrm{~B}=\right.$ niche breadth; $\mathrm{p}_{\mathrm{i}}=$ the proportion of the species which is found in environment $i ; n=$ the number of environmental classes within an experiment). 
situation suggests that females can change their discrimination dependent on the situation.

From the egg production data in the O.S.P. experiments on the different food components, it is possible to calculate rank correlations between all possible pairs of Drosophila species. From the matrix of correlations (Table 5) we constructed an ecological tree of the subgroup using the UPGMA method of Sneath \& Sokal (1973; Spearman's correlations between pairs of species, choice of maximum correlations and matrix shrinking). Figure 5 shows the phenetic distances between the species for the 'no choice' (5A) and 'choice' (5B) experiments. From these ecological trees and the niche breadth calculations (Table 4) it is obvious that oviposition site preference in the 'no choice' situation appears to be the character which furnishes relations which fit best the genetic relations of the species known from the literature. The character presents, firstly the largest range in niche breadths, consequently the best discrimination between the species (Table 4) and secondly, the best similarity with the genetic trees obtained by the analysis of allozyme patterns (Eisses et al., 1979) and the analysis based on polytene chromosome banding sequences (Lemeunier \& Ashburner, 1976). Another interesting fact from Figure 5 is, that Drosophila mauritiana seems to occupy a different position in the ecological trees.

From the literature and our experiments $D$. erecta emerges as somewhat atypical of the subgroup species: the species shows a discrimination between sterol mutant yeasts (Table 2) in contrast to the other species; the species shows a relatively 'narrow niche' in the 'choice' and 'no-choice' experiments for food components (Fig. 3). The fact that the species was collected only from Pandanus candelabrum fruits (Lachoise \& Tsacas, 1974)
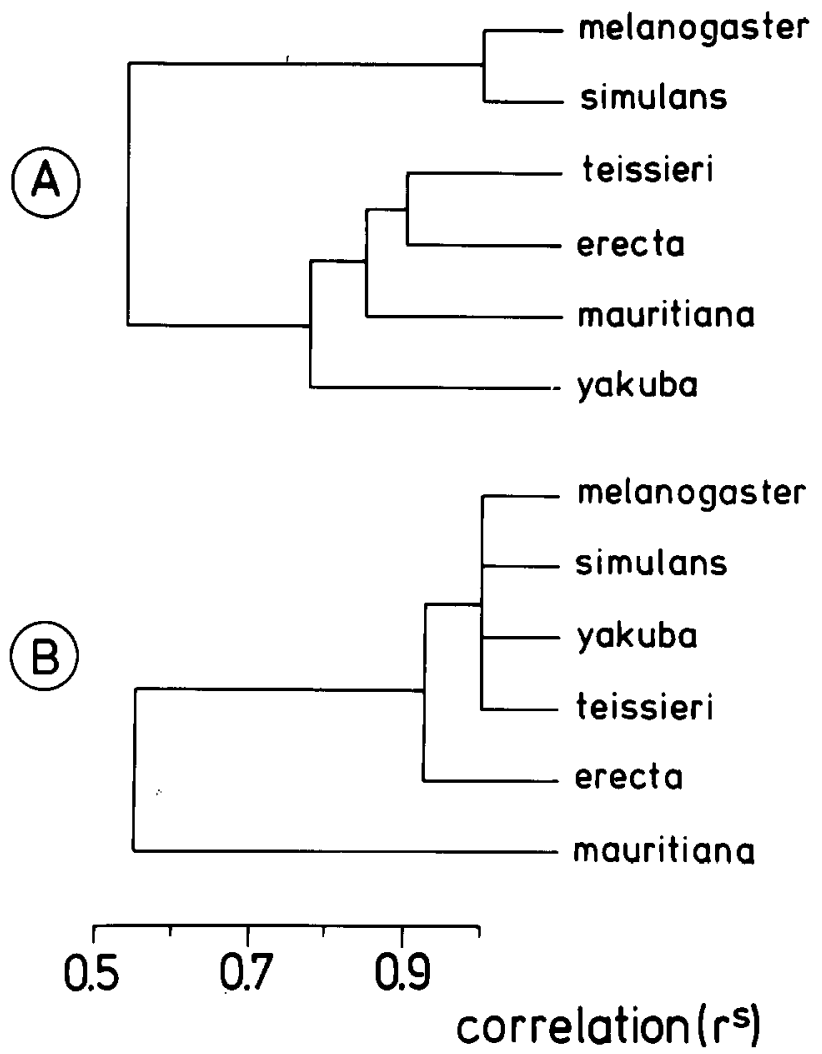

Fig. 5. Ecological trees in the D. melanogaster-subgroup species, based on 'O.S.P.' in a 'no-choice' (a) and 'choice' (b) situation of food components.

suggests a plant specialization caused by sterol dependence. Such a specialization was reported earlier for $D$. pachea by Heed \& Kircher (1965).

The other species we studied do not show yeast preferences (Table 2), but we notice differences in survival on the sterol mutant yeasts (Fig. 4). These developmental differences between the species support the speculation of Dobzhansky (1956) that

Table 5. Spearman's rank correlations between pairs of D. melanogaster subgroup species for egg production on food components.

\begin{tabular}{|c|c|c|c|c|c|c|c|c|c|c|c|c|}
\hline & \multicolumn{6}{|c|}{ 'No-choice' situation } & \multicolumn{6}{|c|}{ 'Choice' situation } \\
\hline & mel. & sim. & teis. & ere. & maur. & yak. & mel. & sim. & teis. & ere. & maur. & yak. \\
\hline mel. & 1 & & & & & & 1 & & & & & \\
\hline sim. & 1 & 1 & & & & & 1 & 1 & & & & \\
\hline teis. & 0.4 & 0.7 & 1 & & & & 1 & 1 & 1 & & & \\
\hline ere. & 0.4 & 0.4 & 0.9 & 1 & & & 0.9 & 0.9 & 0.9 & 1 & & \\
\hline maur. & 0.5 & 0.5 & 0.9 & 0.8 & 1 & & 0.7 & 0.7 & 0.7 & 0.4 & 1 & \\
\hline yak. & 0.6 & 0.6 & 0.9 & 0.8 & 0.7 & 1 & 1 & 1 & 1 & 1 & 0.7 & 1 \\
\hline
\end{tabular}


sibling species largely differentiated adaptively through physiological channels. In the light of these experiments on sterol mutant yeasts it can be argued that since the differences between the species are largely caused by the evident differential mortalities in the pupal or pre-pupal stage on erg-2 (Fig. 6), the third instar larvae of the species differ in their micro sterol requirement for ecdysone production (see also Bos et al., 1976).

Other - circumstantial - evidence of physiological differences between the species is found in the fact that e.g. D. melanogaster and D. erecta immediately and strongly prefer live yeast medium above medium with killed yeast, while e.g. D. mauritiana only develops an increase in egg production during food consumption (Tab. 3). So the species clearly differ in preference for, or rate of use of, yeast media. The importance of yeast as an environmental factor in the selection or regulation of genes in Drosophila enzyme systems has been emphasized by Powell (1970), Thoday (1972), McDonald \& Ayala (1974) and recently by Clarke et al. (1979). Further study of the effects of the physiological conditions of yeast on quantitative characters and/or genetic polymorphisms could present useful data for the analysis of mechanisms involved in the maintenance of genetic variability in Drosophila populations.

\section{Acknowledgements}

Our special thanks are due to Drs Wilke van Delden and Rolf Hoekstra for helpful discussions and comments on the manuscript, to Henk Mulder for drawing the figures and to Pia Scheperkamp, Lammina Krol and Hil Lochorn for careful preparation of the manuscript.

\section{References}

Ali, A. M. M. \& El Helw, M. R., 1974. Differences in the yeasts preferred by Drosophila melanogaster and D. simulans. Egypt. J. Genet. Cytol, 3: 204-210.

Ayala, F. J., Tracey, M., Hedgcock, D. \& Richmond, R. C. 1975. Genetic differentiation within the melanogaster species group of the genus Drosophila (Sophophora). Evolution 28: 576-592.

Beardmore, J. A., Van Delden, W. \& Alkema, L., 1963. A population cage for incubator use. D.I.S. 37: 137.

Begon, M., 1973. Preliminary observations on the yeasts associated with Drosophila obscura and D. subobscura D.I.S. 50: $128-129$.

Bos, M., Burnet, B., Farrow, R. \& Woods, R. A., 1976. Development of Drosophila on sterol mutants of the yeast Saccharomyces cerevisiae. Genet. Res., Camb. 28: 163-176

Bos, M. \& Scharloo, W., 1973. The effects of disruptive and stabilizing selection on body size in Drosophila melanogaster. I. Mean values and variances. Genetics 75: 679-693.

Carson, H. L., 1971. Speciation and the founder principle. Stadler Symp. 3: 51-70.

Clarke, B., Camfield, R. G., Galvin, A. M. \& Pitts, C. R., 1979. Environmental factors affecting the quantity of alcohol dehydrogenase in Drosophila melanogaster. Nature 280: 517-518.

Da Cunha, A. B., 1951. Modification of the adaptive values of chromosomal types in Drosophila pseudoobscura by nutritional variables. Evolution 5: 395-404.

David, J. \& Tsacas, L., 1975. Les Drosophilidae(Diptera) de l'Ile de la Réunion et d'lle Maurice Ill Biologie et origine des espèces. Beitr. Ent. 25: 245-254.

Dobzhansky, Th., 1956. What is an adaptive trait? Am. Nat. 90: 337-347.

Eisses, K. T., Van Dijk, H. \& Van Delden, W., 1979. Genetic differences within the melanogaster species group of the genus Drosophila (Sophophora). Evolution 33: 1063-1068.

Heed, W. B. \& Kircher, H. W., 1965. Unique sterol in the ecology and nutrition of Drosophila pachea. Science 149: $758-761$.

Lachaise, D. \& Tsacas, L., 1974. Les Drosophilidae des savanes pré-forestières de la région tropicale de Lamto(Côte d'Ivoire). II. Le peuplement des fruits de Pandanus candelabrum (Pandanacées). Ann. Univ. Abidjan E. 7: 153-192.

Lemeunier, F. \& Ashburner, H., 1976. Relationships within the melanogaster species subgroup of the genus Drosophila (Sophophora). II. Phylogenetic relationships between six species based upon polytene chromosome banding sequences. Proc. R. Soc. Lond. B. 193: 275-294.

Levins, R., 1968. Evolution in changing environments. Princeton, New Jersey: Princeton University Press.

McDonald, J. F. \& Ayala, F. J., 1974. Genetic response to environmental heterogeneity. Nature 250: 572-574.

McKenzie, J. A. \& Parsons, P. A., 1972. Alcohol tolerance: an ecological parameter in the relative success of Drosophila melanogaster and D. simulans. Oecologia 10: 373-388.

Powell, J. R., 1971. Genetic polymorphisms in a varied environment. Science 174: 1035-1036.

Sneath, P. H. A. \& Sokal, R. R., 1973. Numerical taxonomy. San Francisco, Freeman and Cy.

Thoday, J. M., 1972. Disruptive selection. Proc. R. Soc. Lond. B. 182: 109-143.

Tsacas, L. \& David, J., 1974. Drosophila mauritiana n.sp. du groupe melanogaster del'lle Maurice(Dipt.: Drosophilidae). Bull. Soc. ent. Fr. 79: 42-46.

Tsacas, L. \& Lachaise, D., 1974. Quatre nouvelles espèces de la Côte d'Ivoire du genre Drosophila, groupe melanogaster et discussion de l'origine du sous-groupe melanogaster (Diptera: Drosophilidae). Ann. Univ. Abidjan E. 7: 193-211.

Received 10.2.1981. Accepted 6.5.1981. 